\title{
Biosíntesis y caracterización de nanoestructuras por microscopía electrónica ${ }^{\diamond}$
}

\section{Biosynthesis and characterization of nanostructures by electron microscopy}

\author{
Nayely Torres-Gómez, ${ }^{*}$ Rodolfo D. Ávila-Avilés, ${ }^{* *}$ Alfredo R. Vilchis-Nestor ${ }^{* * *}$
}

\begin{abstract}
The methods to obtain nanostructures have been a fundamental area of study since the beginning of the nanotechnology. The biosynthesis of nanostructures is inspired by the behavior that some microorganisms and plants have for subsisting in environments with chemical stress. This paper presents newfangled examples of biosynthesis achieved by our research group in order to obtain Ag and Au nanostructures with tunable morphology using Anemopsis californica extract as reducing agent of $\mathrm{Ag}^{+1}$ and $\mathrm{Au}^{+3}$ ions; as well the synthesis assisted by Camellia sinensis extract of magnetite nanoparticles $\left(\mathrm{Fe}_{3} \mathrm{O}_{4}\right)$ with homogeneous size and an excellent crystalline phase control in contrast with other biogenic methods reported in the literature. Additionally, the effective use of biotemplates to design nanostructured materials with green synthesis methods is demonstrated. Finally, the evidence of the nanomaterials generated from biosynthesis was shown via characterization with transmission electron microscopy, scanning electron microscopy and X-ray energy dispersion spectroscopy.
\end{abstract}

KEYWORDS: biosynthesis, Camellia sinensis, Anemopsis californica, Ag, $\mathrm{Au}$ and $\mathrm{Fe}_{3} \mathrm{O}_{4}$ nanoparticles, biotemplate, electron microscopy.

RESUMEN: Los métodos de obtención de nanoestructuras han sido un área de estudio fundamental desde el inicio de la nanotecnología. La biosíntesis de nanoestructuras se inspira en los mecanismos que tienen algunos microrganismos y plantas para subsistir en ambientes con estrés químico. En el presente trabajo se exhiben ejemplos novedosos de biosíntesis que han logrado nuestro grupo de investigación con el fin de obtener nanoestructuras de Ag y Au con morfología fácilmente sintonizable a partir del extracto de hierba del manso (Anemopsis californica) como agente reductor de los iones $\mathrm{Ag}^{+1} \mathrm{y} \mathrm{Au}^{+3}$; así como la síntesis asistida con el extracto de té verde (Camellia sinensis) de nanopartículas de magnetita $\left(\mathrm{Fe}_{3} \mathrm{O}_{4}\right)$ con tamaño homogéneo y un excelente control de fase cristalina en comparación con lo reportado en la literatura con

Recibido: 25 de febrero de 2020.

Aceptado: 22 de abril de 2020.

${ }^{\diamond}$ Agradecemos a los proyectos Conacyt No. 280518 y No. A1-S-34533, por el apoyo financiero otorgado.

* Tecnológico Nacional de México/Campus Toluca. División de Posgrado e Investigación. Av. Tecnológico s/n Ex Rancho "La Virgen”, CP 52140, Metepec, Estado de México, México. Tel. 7224068112. Correo electrónico: nash.togoz@gmail.com

** Centro de Investigación y Estudios Avanzados del Instituto Politécnico Nacional (CINVESTAVIPN), Departamento de Genética y Biología Molecular. Avenida Instituto Politécnico Nacional 2508, La Laguna Ticoman, CP 07360, Ciudad de México, México. Tel. +52-55-5747-3800 Ext. 5360. Correo electrónico: rodolfo.avila@cinvestav.mx

*** Centro Conjunto de Investigación en Química Sustentable (CCIQS), UAEM-UNAM. Km 14.5, Toluca-Atlacomulco Road, Toluca, Estado de México, México, CP 50200. Tel. +521-7222766610 Ext. 7712.

Autor de correspondencia: arvilchisn@uaemex.mx 
métodos biogénicos. Adicionalmente, se demuestra el uso efectivo de biotemplates para diseñar materiales nanoestructurados con métodos de síntesis verde. Por último, se presenta evidencia de las características estructurales, morfológicas y químicas de los nanomateriales generados a partir de biosíntesis con base en la caracterización con microscopía electrónica de transmisión, microscopía electrónica de barrido y espectroscopía de dispersión de energía de rayos-X.

PALABRAS CLAVE: biosíntesis, Camellia sinensis, Anemopsis californica, nanopartículas de Ag, $\mathrm{Au}$ y $\mathrm{Fe}_{3} \mathrm{O}_{4}$, biotemplate, microscopía electrónica.

\section{Introducción}

Los materiales con tamaño nanométrico poseen propiedades físicas y químicas diferentes a su contraparte "en bulto" asociadas con fenómenos que se presentan solo en la escala del orden de los nanómetros. Estas propiedades hacen que las nanoestructuras tengan un alto potencial para resolver problemas en áreas como la medicina, microelectrónica, remediación ambiental, desarrollo de sensores y catálisis. Dichas propiedades están íntimamente ligadas con las características (forma, tamaño, fase cristalina, composición química y arreglo estructural) de las nanoestructuras (Schmid, 1992; Kelly et al., 2003)

Por esta razón, el desarrollo de metodologías para la síntesis direccionada de nanoestructuras con un control adecuado sobre las características de estas, continúa siendo uno objetivo fundamental de la nanotecnología. A la fecha se han logrado fabricar nanopartículas con una morfología bien definida (esféricas, hexagonales, triangulares, etc.), con un rango de tamaño estrecho y con una composición química homogénea (Sajanlal et al., 2011; Xia et al., 2013; Pileni, 2007) a través de métodos de síntesis químicos (Sau y Rogach, 2010) y físicos (Mohanpuria, Rana y Yadav, 2008), tales como la reducción química, la reducción electroquímica, la fotorreducción, la ablación láser, la evaporación térmica, entre otros. Sin embargo, el desarrollo de una metodología de síntesis eficiente, económica y "eco-amigable" permanece como un desafío, ya que las metodologías anteriores implican el uso altas cantidades de energía, equipo costoso y/o reactivos químicos tóxicos.

La biosíntesis también llamada síntesis "verde" (Mohanpuria, Rana y Yadav, 2008) se basa en el uso de microrganismos (bacterias y hongos) (Bhattacharya y Gupta, 2005), plantas vivas (Gardea-Torresdey et al., 2003) y extractos de plantas (Iravani, 2011) para obtener nanoestructuras. La síntesis verde ha emergido en las últimas décadas como una alternativa a procedimientos sintéticos de tipo químico o físico, debido a que tiene como ventajas la eliminación de solventes orgánicos, agentes reductores y/o estabilizantes amigables con el ambiente, no se necesitan altos consumos de energía ni equipos sofisticados; además de que las reacciones se llevan a cabo en condiciones ambientales de presión, temperatura y $\mathrm{pH}$.

Sin embargo, la síntesis de nanopartículas asistida con rutas biogénicas tiene como retos importantes el de conseguir un control morfológico y de tamaño al menos comparable con los métodos físicos y químicos, así como ge- 
nerar arreglos nanométricos con la dimensionalidad deseada. Adicionalmente el abanico de nanopartículas que se obtienen por biorreducción ha estado limitado a elementos con un Potencial Estándar de Reducción positivo como el $\mathrm{Au}\left(\mathrm{E}^{\circ}=1.4 \mathrm{~V}\right)$ y la $\mathrm{Ag}\left(\mathrm{E}^{\circ}=0.8 \mathrm{~V}\right)$, (Yoosaf et al., 2007) dejando fuera materiales de amplio interés en optoelectrónica y catálisis como lo son los óxidos y calcogenuros metálicos. Aunado a esto un desafío importante es la comprensión profunda de los mecanismos implicados en la biosíntesis de nanopartículas. La complejidad del sistema bioquímico hace que la identificación de las especies activas en la nucleación, crecimiento y estabilización de las nanoestructuras sea bastante difícil.

Las ventajas que ofrecen las rutas verdes de nanopartículas y sus retos asociados han convertido a la biosíntesis en un área de investigación con un considerable potencial de desarrollo, como lo demuestra el número creciente de publicaciones que se generan sobre el área a pesar de ser relativamente nueva. Una revisión breve del estado del arte de los procesos de síntesis verde para generar nanopartículas se puede explorar en los trabajos de (Kumar y Sudesh, 2009); (Sanchez-Mendieta y Rafael, 2012); (López et al., 2013); (Quester, Avalos-Borja y Castro-Longoria, 2013).

La biosíntesis de nanomateriales había sido ya observada por los microbiólogos al estudiar un grupo de microrganismos llamado "extremófilos". Particularmente las bacterias y los hongos a menudo están expuestos a condiciones ambientales extremas (temperaturas altas o bajas, $\mathrm{pH}$ altos o altas concentraciones de sales), por lo que para sobrevivir deben tener una estrategia para resistir ese estrés medioambiental. Cuando los microrganismos son expuestos a toxicidad por altas concentraciones de iones metálicos despliegan mecanismos de defensa que incluyen sistemas de flujo, alteraciones de la solubilidad y toxicidad, modifican el estado de oxidación de iones metálicos formando complejos, o precipitando los metales intracelularmente, así como bloqueando sistemas de transporte para un metal en específico. Estos bioprocesos son la base de la biolixiviación, la biorremediación, la corrosión microbiana, así como la biomineralización y la síntesis de nanopartículas.

El uso de organismos eucariontes, como los hongos, tiene mucho potencial para la biosíntesis de nanopartículas debido a que son fáciles de manejar en el laboratorio y secretan grandes cantidades de proteínas, que son las biomoléculas asociadas con la formación de nanoestructuras en estos sistemas. Además, se trata de procesos fácilmente escalables y económicamente viables, con la posibilidad de cubrir fácilmente grandes áreas de producción con un adecuado crecimiento de los micelios. En nuestro grupo de investigación se ha demostrado la versatilidad de los hongos para generar nanopartículas mono y bimetálicas de Au-Ag cuando se utiliza N. crassa. (Castro-Longoria et al., 2011) Estudios posteriores demuestran que es posible favorecer crecimientos anisotrópicos dependiendo del tipo de hongo utilizado, como cuando se ocupa B. Cinerea para obtener nanoestructuras 2D de Au (figura 1). 
Figura 1. Nanopartículas triangulares de Au obtenidas con B. cinerea.

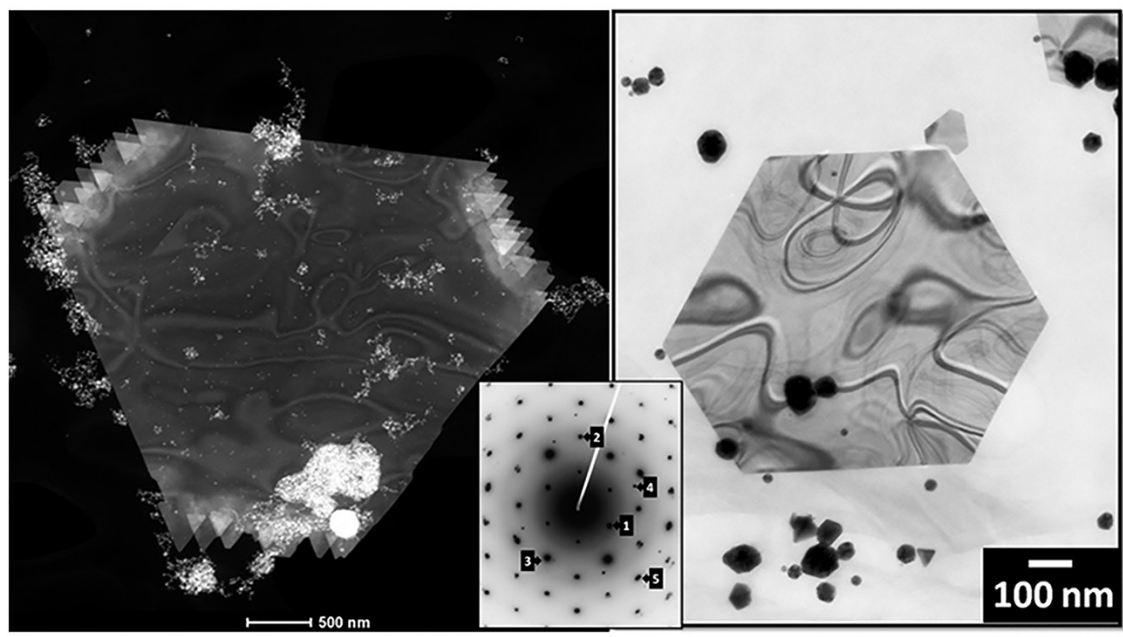

Fuente: Imagen de contraste Z, cortesía del Dr. Miguel Ávalos-Borja, IPICYT.

Por otra parte, el empleo de plantas para la biosíntesis de nanopartículas tiene ventajas sobre los métodos que emplean microrganismos: no es necesario mantener cultivos celulares y no se requieren procesos de separación y/o purificaciones posteriores. Los procesos biosintéticos para nanopartículas son más útiles cuando se producen de forma extracelular, como sucede cuando se emplean extractos de plantas. Adicionalmente, su tamaño, dispersión y morfología se pueden controlar de forma más rápida y simple, además, el uso de plantas resulta adecuado para escalar a nivel industrial la síntesis de nanopartículas.

En el presente artículo se reporta la biosíntesis de nanopartículas de $\mathrm{Au}$, $\mathrm{Ag}$ y $\mathrm{Fe}_{3} \mathrm{O}_{4}$ asistida con los extractos Anemopsis californica y Camellia sinensis, respectivamente. Así como su caracterización por microscopía electrónica y técnicas espectroscópicas.

\section{Procedimiento experimental}

\section{Síntesis de nanopartículas de oro y plata usando extracto}

de A. californica

El nitrato de plata $\left(\mathrm{AgNO}_{3}\right)$ y el ácido tetracloroáurico $\left(\mathrm{HAuCl}_{4} \cdot 3 \mathrm{H}_{2} \mathrm{O}\right)$ fueron proporcionados por Sigma Aldrich Chemials, y fueron usados como se recibieron. Los solventes como metanol e isopropanol, ambos en grado reactivo, fueron obtenidos de Fermont Company y se usaron sin ninguna purificación. Se empleó agua desionizada en todos los pasos de las síntesis.

Las hojas de A. californica fueron obtenidas de Ensenada, México. Para el proceso de extracción se secaron a temperatura ambiente, posteriormente 
fraccionadas con el propósito de incrementar la superficie de contacto, acto seguido se lavaron con agua desionizada para limpiarlas. El extracto acuoso de $A$. californica fue preparado mezclando la biomasa en agua desionizada a punto de ebullición por dos minutos, después se dejó enfriar a temperatura ambiente hasta llegar a $20^{\circ} \mathrm{C}$ y filtrado para remover la biomasa. La misma metodología fue ocupada para preparar los extractos con metanol e isopropanol.

Las nanopartículas de plata fueron sintetizadas utilizando los extractos con diferente solvente (agua, metanol e isopropanol, respectivamente) de la siguiente manera: el de extracto de $A$. californica fue añadido a una solución acuosa $10^{-3} \mathrm{M}$ de nitrato de plata. Tras 24 hrs de reacción las nanopartículas fueron caracterizadas. La misma metodología fue utilizada para la síntesis de nanopartículas de oro a partir de una solución $10^{-3} \mathrm{M}^{\mathrm{de}} \mathrm{HAuCl}_{4}$.

\section{Síntesis de nanopartículas de óxidos metálicos con Camellia sinensis}

Los reactivos se usaron como se suministran, sin posterior purificación. Sulfato férrico amónico dodecahidratado $\left(\mathrm{Fe}\left(\mathrm{SO}_{4}\right) 2 \mathrm{NH}_{4} \cdot 12 \mathrm{H}_{2} \mathrm{O}\right)$, hidróxido de amonio ACS al 29\% $\left(\mathrm{NH}_{4} \mathrm{OH}\right)$ marca Fremont, alcohol etílico al 96\% $\left(\mathrm{CH}_{3} \mathrm{CH}_{2} \mathrm{OH}\right)$ marca Golden Bell, té verde marca Lagg's y agua desionizada.

Para la preparación del extracto se pesaron y homogenizaron en mortero de ágata 2 gramos de té verde (Camellia sinensis) marca Lagg's, el cual fue agregado a $20 \mathrm{ml}$ de etanol $\left(\mathrm{T}=60^{\circ} \mathrm{C}\right)$, y se mantuvo en agitación por 10 min. Dejándose enfriar a temperatura ambiente y filtrándose al vacío. Las nanopartículas de óxidos de hierro fueron preparadas como sigue: se agregó $\mathrm{NH}_{4} \mathrm{OH}$ al 29\% en $34 \mathrm{ml}$ de agua desionizada, la solución "se sónico" por 5 minutos en un equipo Branson 2500, después se adicionó gota a gota una solución $0.1 \mathrm{M}$ de $\mathrm{Fe}\left(\mathrm{SO}_{4}\right) 2 \mathrm{NH}_{4} \cdot 12 \mathrm{H}_{2} \mathrm{O}$, "se sónico" 20 minutos, enseguida se agregó el extracto de té verde $(0.5,1$ y $2 \mathrm{ml}$; las cuales se asignaron con la notación F1, F2 y F3, respectivamente). La temperatura y el pH de la reacción se registraron después de cada intervalo de sonicación. Transcurrido el tiempo de síntesis, cada reacción se dejó enfriar a temperatura ambiente. El precipitado obtenido se lavó varias veces con etanol. Posteriormente se secó en una estufa por 30 minutos a una temperatura de $70{ }^{\circ} \mathrm{C}$, para su ulterior caracterización.

\section{Sintesis de nano/microestructuras híbridas Ag-Fe304 usando como templete granos de polen}

Para la síntesis de las nano/microestructuras híbridas, el nitrato de plata $\left(\mathrm{AgNO}_{3}\right)$, sulfato férrico amónico dodecahidratado $\left(\mathrm{Fe}\left(\mathrm{SO}_{4}\right) 2 \mathrm{NH}_{4} \cdot 12 \mathrm{H}_{2} \mathrm{O}\right)$, y el hidróxido de amonio ACS al $29 \%\left(\mathrm{NH}_{4} \mathrm{OH}\right)$ marca Fremont fueron utilizados como se suministran. Para la síntesis se ocuparon de granos de polen

${ }^{1}$ N. del E. de sonicación: acto de aplicación de energía del sonido (generalmente ultrasonido), para agitar partículas de muestra con diversos fines científicos e industriales. 
recién colectado y mezclado en una solución $0.1 \mathrm{M}$ de $\mathrm{AgNO}_{3}$, mantenido en agitación magnética durante cuatro horas. Luego la solución fue filtrada al vacío ocupando un filtro de nitrocelulosa de 1.2 micrómetros, las nano/microestructuras fueron recuperadas y calcinadas a $180{ }^{\circ} \mathrm{C}$ durante $12 \mathrm{~h}$. Después de esto se colocaron los granos de polen funcionalizados con las nanopartículas de plata con una solución $\mathrm{FeSO}_{4} 0.1 \mathrm{M}$, y se añadió $\mathrm{NH}_{4} \mathrm{OH}$ por goteo manteniendo la agitación por 72 horas. Acto seguido, las nano/microestructuras híbridas fueron recuperadas por filtración y caracterizadas.

\section{Caracterización}

Microscopía electrónica de transmisión:

Cinco microlitros de cada muestra de nanopartículas (AgNPs, $\mathrm{AuNps}_{\mathrm{FFe}} \mathrm{O}_{4} \mathrm{NPs}$ ) fueron puestos en una rejilla de cobre (SPI-supplies) previamente cubierta por una capa fina de Formvar y recubierta con grafito mediante la deposición con un Sputtering DENTON a 30 mTorr de presión. Por otra parte, para caracterizar la formación de nanopartículas de plata funcionalizadas en granos de polen, la nano/microestructura fue embebida en resina epóxica, permitiendo la polimerización incubando a $60^{\circ} \mathrm{C}$ por 24 horas. Secciones ultradelgadas de $100 \mathrm{~nm}$ de la muestra fueron obtenidas utilizando un ultramicrotomo (Leica Ultracut R) y más adelante puestas en rejillas de cobre cubiertas con carbono (SPI-supplies). Las muestras de $\mathrm{Au}, \mathrm{Ag}$ y Ag- $\mathrm{Fe}_{3} \mathrm{O}_{4} /$ polen fueron analizadas con un microscopio de transmisión (TEM) JEOL-2100 operado con una aceleración de voltaje de $200 \mathrm{kV}$ y equipado con dos detectores para STEM, de campo claro (BF-detector) y anular de campo oscuro (ADF-detector). Las nanopartículas de $\mathrm{Fe}_{3} \mathrm{O}_{4}$ se caracterizaron por TEM con el uso de un FEI TEM Titan G2 80-300 operado a 300 kV, con capacidades de microscopía electrónica de transmisión de exploración (STEM), y equipado con un campo oscuro anular de alto ángulo (HAADF) detector de Fishione; un detector STEM de campo brillante de Gatan; un detector ADF STEM de Gatan; y un detector de espectroscopía de rayos X con dispersión de energía EDAX (EDXS). Las micrografías TEM, HRTEM, SAED y STEM ADF fueron analizadas usando el software Gatan Digital Micrograph TM y el software ImageJ para determinar el tamaño de partícula. Para los análisis realizados con HRTEM y SAED, los datos experimentales fueron indexados con base en la carta JCPDS 0-004-0783 para plata, 00-004-0784 para oro y 19-0629 para $\mathrm{Fe}_{3} \mathrm{O}_{4}$, respectivamente. Para determinar la polidispersidad de las nanopartículas se consideraron más de 500 nanopartículas para la medición en cada muestra.

Microscopía electrónica de barrido:

Las nano/microestructuras híbridas tras la síntesis fueron montadas sobre cinta de cobre para su análisis por microscopía electrónica de barrido y análisis de dispersión de energía (EDS) acoplado. Las muestras fueron analizadas utilizando un microscopio electrónico de barrido JEOL JSM-6510LV, con un voltaje de aceleración de $20 \mathrm{kV}$ para obtener las micrografías y $15 \mathrm{kV}$ para los EDS, usando los detectores de electrones secundarios y retrodisper- 
sos, en modalidad de alto vacío. El análisis químico elemental por EDS se llevó a cabo empleando un detector de rayos X de la marca Bruker modelo QUANTAX 200 y con resolución de 129 eV acoplado al microscopio.

\section{Espectroscopía Raman:}

Para realizar las mediciones Raman, las muestras fueron preparadas con el siguiente protocolo: las nanopartículas de $\mathrm{Fe}_{3} \mathrm{O}_{4}$ se dispersaron en isopropanol y se coloca una gota de esta dispersión sobre un portaobjeto de vidrio dejándola secar a temperatura ambiente. Las muestras se analizaron en un sistema microRaman, marca Jobin Yvon Horiba, modelo LabRam HR800, con láser monocromático de $632.8 \mathrm{~nm}$, obj x 50 , una potencia de $0.7 \mathrm{~mW}$ con 10 adquisiciones por 60 segundos, rejilla de 600 líneas $/ \mathrm{mm}$ y una apertura de $400 \mu \mathrm{m}$.

\section{Difracción de rayos $\mathrm{X}$ :}

Las nanopartículas de $\mathrm{Fe}_{3} \mathrm{O}_{4}$ fueron analizadas con difractómetro de polvos Bruker D8 Advance con geometría Bragg-Bretano, radiación de $\mathrm{CuK \alpha}$, y detector Linxeye. El equipo tiene capacidad para determinar el tamaño de cristalita y análisis de fases con la ayuda del programa PDF-2 conectado a la base CCDB. Fuente de rayos X: tubo de descarga con ánodo de cobre $(\mathrm{K} \alpha 1=$ $0.1540 \AA$ ).

\section{Resultados y discusión}

\section{Síntesis de nanopartículas de oro y plata usando extracto}

\section{de A. californica}

La síntesis de nanopartículas de metales nobles ( $\mathrm{Ag}$ y $\mathrm{Au})$ mediante la utilización de extractos de Anemopsis californica ha sido recientemente explorada por nuestro grupo de investigación. A. californica comúnmente conocida como hierba del manso o hierba mansa, es una planta nativa del suroeste de Estados Unidos y el norte de México. Estudios de composición de extractos de A. californica revelan la presencia demetil eugenol, timol, piperitona, éter timol metilo, R-pineno, 1,8-cineol, metilchavicol, y p-cimeno (Medina et al., 2005) los cuales pueden servir como agentes reductores y estabilizadores en la síntesis de las nanopartículas metálicas. Del-Toro Sánchez y colaboradores reportaron y determinaron la presencia de fenoles y flavonoides totales, tanto en hojas, tallos y raíces de la planta, determinando la presencia de fenoles totales en $27 \mathrm{mg}$ por gramo de biomasa seca y de $16 \mathrm{mg}$ por gramo de biomasa para flavonoides en promedio en las hojas A. californica (Del-Toro-Sánchez et al., 2015). Por otra parte, ha sido ampliamente reportado que flavonoides y fenoles son los encargados de la formación de nanopartículas de oro y plata gracias a la reducción de las sales precursoras; es así como proponemos que la presencia de dichos compuestos son los encargados de la síntesis de las nanopartículas biogénicas obtenidas por nuestro grupo de trabajo (Iravani, 2011). 
Con la utilización de extractos de hojas de A. californica, se han obtenido nanopartículas metálicas que muestran distribuciones diferentes, así como diversas características en tamaño y forma. En el caso del uso de extractos acuosos se han obtenido nanoestructuras de plata con morfologías variadas como rodillos, inukshuks y flores (figura 2A, 2B, y 2C, respectivamente) de tamaños variables; en contraste con la obtención de nanopartículas poliédricas de plata obtenidas mediante el uso de extractos en base metanol e isopropanol; con un tamaño promedio que ronda los $23 \pm 13.2 \mathrm{~nm}$ (figura $3 \mathrm{~A}$ ).

Figura 2. Nanoestructuras de plata obtenidas con extracto acuoso de A. californica: A) rodillos (STEM$A D F) ; B$ ) inukshuks (STEM-ADF), y, C) flores (STEM-ADF).
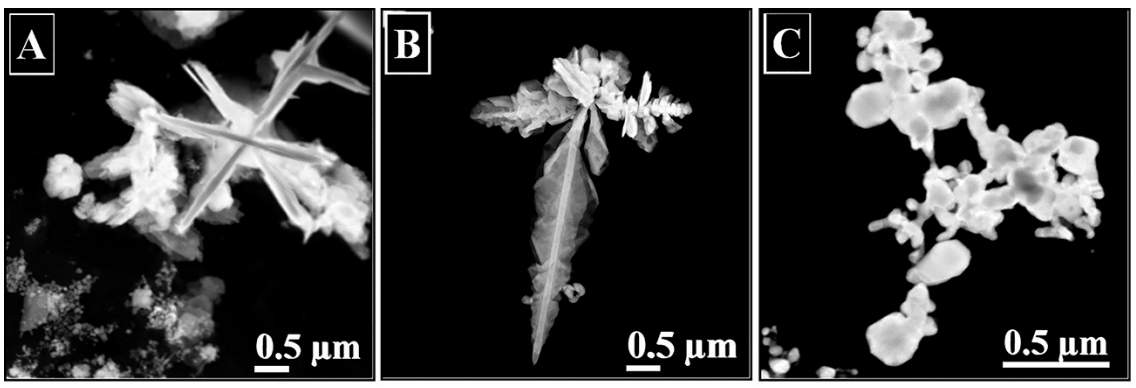

Fuente: Elaboración de los autores.

El patrón de difracción de electrones (SAED) revela la naturaleza policristalina de las nanopartículas de Ag (figura $3 \mathrm{E}$ ) cuyos anillos de difracción se pueden indexar con base en la estructura FCC de la plata como (111) (220) y (200), respectivamente. La distancia interplanar obtenida por HRTEM (figura 3 C) es consistente también con la estructura cúbica centrada en caras de la plata.

En el caso de las nanopartículas de oro, estas se obtuvieron con dos morfologías prevalentes: poliédricas y planas triangulares. El extracto acuoso de A. californica permite obtener nanopartículas poliédricas de un tamaño promedio de $40 \pm 30.23 \mathrm{~nm}$, que es mayor al tamaño de las nanopartículas generadas con los extractos base metanol e isopropanol que tienen tamaños promedio de $20 \pm 15.23$ y $10 \pm 7.9 \mathrm{~nm}$, respectivamente (figura 3B). La formación de nanopartículas triangulares planas solo fue posible mediante el uso de extractos base agua y metanol, pero no con isopropanol. La imagen HRTEM en la figura 3D confirma la presencia de los planos (100) y (200) consistentes con la estructura FCC del Au. El patrón SAED de la figura 3F sugiere que el sistema es policristalino, aunque se observan puntos de difracción bien definidos que pueden estar asociados con las nanopartículas triangulares planas orientadas en la dirección [111] normal a la superficie de una de sus caras planas con respecto al haz de electrones. 
Figura 3. Imágenes de TEM y patrones SAED representativas, las nanopartículas obtenidas con el extracto acuoso de $A$. californica de $\mathrm{Ag}(\mathrm{A}, \mathrm{C}, \mathrm{E})$ y $\mathrm{Au}(\mathrm{B}, \mathrm{D}, \mathrm{F})$, respectivamente.
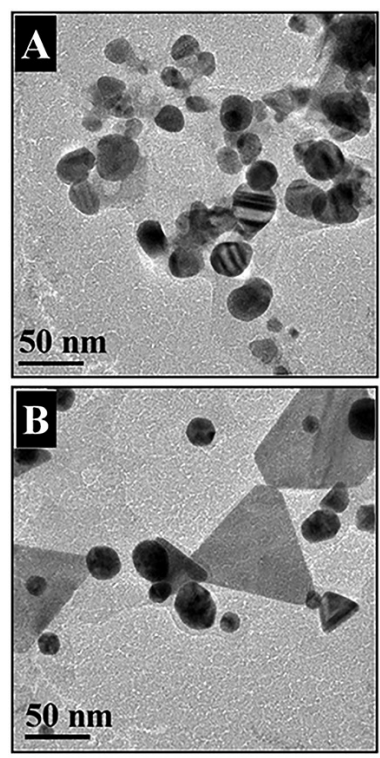

Fuente: Elaboración de los autores.
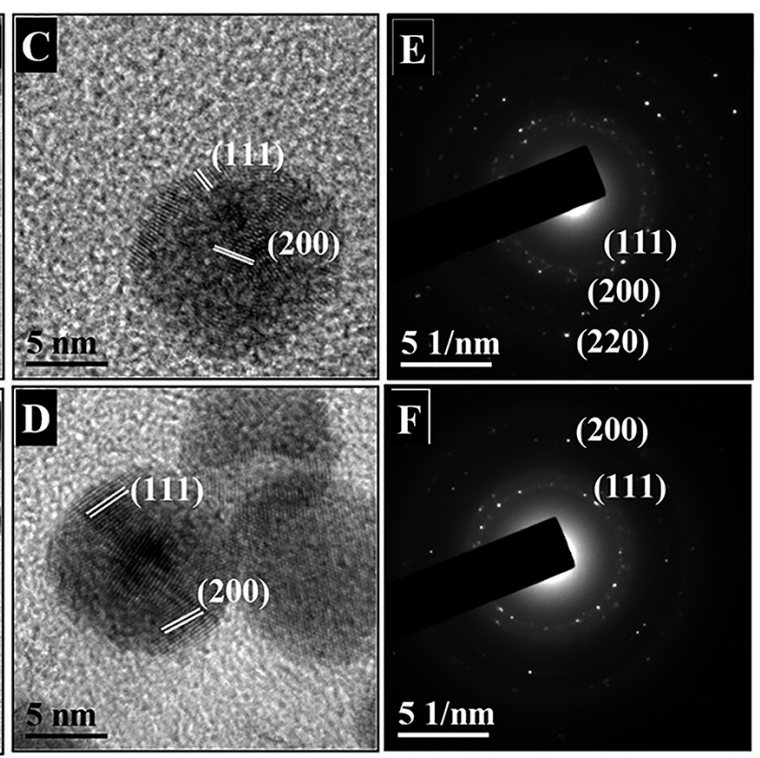

Síntesis de nanopartículas de óxidos metálicos con Camellia sinensis Los óxidos metálicos nanoestructurados se han estudiado debido a las propiedades que presentan en la escala nanométrica. Entre estos materiales se encuentran los óxidos de hierro, principalmente la magnetita, que, al estar en tamaños menores de $20 \mathrm{~nm}$, presenta superparagmetismo, ampliamente utilizado en aplicaciones biológicas, por ejemplo, en el posible tratamiento contra el cáncer. Existen muchos métodos de síntesis para obtener nanopartículas de $\mathrm{Fe}_{3} \mathrm{O}_{4}$ siendo los químicos los que dan mejor resultado en control de tamaño y morfología. La eficacia del extracto de Camellia sinensis para sintetizar nanopartículas de $\mathrm{Ag}$ y $\mathrm{Au}$ fue reportado por primera vez por nuestro grupo de investigación (Vilchis-Nestor et al., 2008), y desde entonces se ha explorado el potencial del extracto de Camellia sinensis como alternativa para controlar el tamaño de varios tipos de nanopartículas gracias al contenido de polifenoles presentes en el extracto.

El extracto acuoso de Camellia sinensis contiene una variedad grande de polifenoles como flavonoides (catecol, epicatequina y cafeína) así como ácidos fenólicos (ácido 3,4-dihidroxibenzoico, ácido vanílico y ácido benzoico). Estos componentes contribuyen a la actividad antioxidante del extracto de té verde y están directamente relacionados con la formación y estabilización de nanopartículas. La extracción con agua alcanza $59.8 \pm 1.8 \mathrm{mg}$ de CAE/g dw (miligramos de $\mathrm{CAE}$ = equivalentes de ácido clorogénico/ dw: gramos del té verde seco) lo que le confiere una capacidad antioxidante de $70.1 \pm 2.4 \%$ (Rababah, 
Hettiarachchy y Horax, 2004) y con ello amplias posibilidades para ser empleado en la biosíntesis de nanopartículas (Loo et al., 2012). Se ha demostrado que la presencia de grupos carboxilo influye directamente en la estabilización de las nanopartículas (Yoosaf et al., 2007) e incluso en la formación de agregados coloidales que ya han sido utilizados eficazmente para sensar iones metálicos en agua (Silva-De Hoyos et al., 2019).

En la figura 4A se muestran los espectros Raman de todas las muestras sintetizadas. Los espectros se obtuvieron con un láser de $632.8 \mathrm{~nm}$ y una potencia de $0.7 \mathrm{~mW}$, con la finalidad de no afectar la muestra y evitar que ocurra un cambio de fase por un aumento de temperatura generada por el láser. Olga N. Shebanova y Peter Lazor (Shebanova y Lazor, 2003) reportaron el efecto de la potencia del láser en el proceso de oxidación de la magnetita al ser analizada por espectroscopía Raman, y explican que se puede realizar una asignación errónea de las bandas que aparecen en los espectros. Considerando esto, podemos observar que las bandas características de la hematita que se encuentran alrededor de $300-410 \mathrm{~cm}^{-1}$ no aparecen en los espectros de las muestras analizadas. Todos los espectros exhiben una banda ancha centrada en $660 \mathrm{~cm}^{-1}$; asignada al enlace $\mathrm{Fe}-\mathrm{O}$ de los sitios tetraédricos de magnetita.

Difracción de rayos $\mathrm{X}$ de polvos (DRX) es una técnica de gran utilidad cuando se pretende identificar alguna fase cristalina; nuestros productos mostraron cristalinidad y los resultados se correlacionan con lo mostrado en los espectros Raman; donde la fase cristalina presente corresponde a la magnetita. Los difractogramas fueron indexados y se compararon con la tarjeta cristalográfica JPCDS 19-0629 de la magnetita. En la figura 4B se presentan los difractogramas de las tres muestras sintetizadas de magnetita en donde el pico más intenso corresponde a la reflexión del plano (311). Los picos indexados corresponden a los planos (220), (311), (400), (422), (511) y (440) de la magnetita, que tiene una estructura cristalina cúbica centrada en las caras (FCC) con parámetro de red $\mathrm{a}=8.393 \AA$ A . No se encontró ningún pico correspondiente a la maghemita, por lo cual podemos confirmar los resultados obtenidos por Raman y decir que no hay impurezas de otra fase cristalina de hierro ni de los precursores en los productos obtenidos.

En la figura 4C pueden observarse las micrografías de TEM de las nanopartículas sintetizadas F1, F2 y F3, con los diferentes volúmenes de extracto (0.5, 1 y 2 ml). En las micrografías de TEM de la muestra F1, F2 y F3 se observa que la morfología prevaleciente es la esférica y la poliedral, con tamaños promedio de $15.36 \pm 4.62 \mathrm{~nm}, 1.72 \pm 0.38 \mathrm{~nm}$ y $21.03 \pm 5.60 \mathrm{~nm}$, respectivamente.

Se obtuvieron patrones de difracción de electrones de área selecta (SAED) y se observa que las nanopartículas son policristalinas. Se indexaron los SAED de las tres muestras y se encontraron los planos (311), (220), (111), correspondientes a la fase de magnetita (figura 4D). 
Figura 4. Caracterización de las nanopartículas de $\mathrm{Fe}_{3} \mathrm{O}_{4}$ obtenidas con $0.5,1$ y $2 \mathrm{ml}$ de extracto de $\mathrm{Ca}$ mellia sinensis (F1, F2 y F3, respectivamente). A) Espectros Raman. B) Patrones de difracción de polvos. C) Micrografias de TEM. D) Patrones de difracción de electrones (SAED).
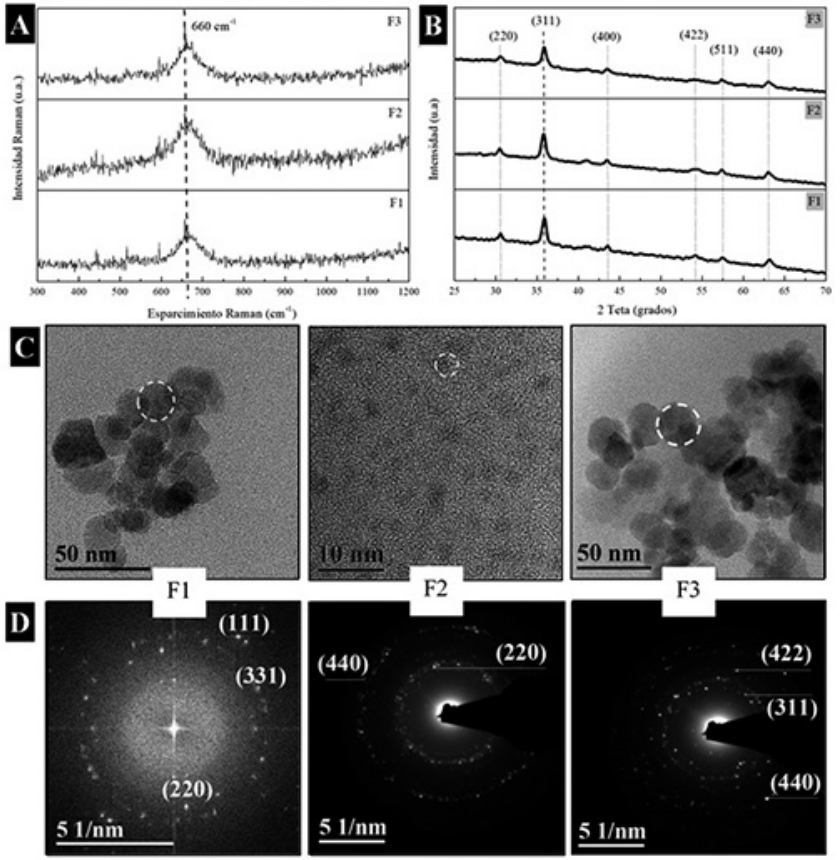

Fuente: Elaboración de los autores.

Sintesis de nano/microestructuras hibridas $\mathrm{Ag}-\mathrm{Fe}_{3} \mathrm{O}_{4}$ usando como templete granos de polen

La naturaleza provee innumerables fuentes de inspiración para el diseño de partículas con morfologías complejas. En este tenor, la generación de partículas bioinspiradas permite el enriquecimiento de las funciones y promoción de las propiedades buscadas. Por tal motivo, hemos implementado la utilización de granos de polen de la planta Dimorphotheca ecklonis como templete para la deposición de nanopartículas, permitiendo que las propiedades de las nanopartículas que lo funcionalizan se vean incrementadas y desarrolladas, debido al confinamiento de las mismas y a la morfología del templete. Por su parte, los granos de polen de Dimorphotheca ecklonis que hemos propuesto pueden ser utilizados en la formación de las nanopartículas de plata. Las nanopartículas pueden ser formadas gracias a la composición de la exina presente en el polen, la cual contiene compuestos aromáticos, fenilpropanoides, polifenoles y carotenoides que actúan como agentes reductores de las sales precursoras en la biosíntesis de nanopartículas (Scott, 1994).

Así, hemos logrado la obtención de nanopartículas de plata funcionalizando granos de polen de Dimorphotheca ecklonis, utilizando como agente re- 
ductor y estabilizador los mismos granos. Aunado a esto, hemos decorado dichas estructuras con nanopartículas de magnetita (obtenidas por el método de precipitación) generando nano/microestructuras híbridas $\mathrm{Ag}-\mathrm{Fe}_{3} \mathrm{O}_{4}$ (figura 5) sin modificar la estructura característica de los granos de polen como se puede observar en las figuras 5A y 5D. Por su parte, las nanopartículas de plata que conforman las nano/microestructuras rondan en un tamaño promedio de $9.37 \pm 6.10 \mathrm{~nm}$ con una morfología esférica, mostrando una distribución homogénea dentro del grano de polen (figura 5B), mientras que las nanopartículas de magnetita tienen un rango de tamaño entre 12 a $14 \mathrm{~nm}$ (figura 5C).

Figura 5. A) Micrografía SEM de nano/microestructuras híbridas $\mathrm{Ag}-\mathrm{Fe}_{3} \mathrm{O}_{4}$ usando como templete granos de polen. B) micrografía TEM de corte de grano de polen funcionalizado con nanopartículas de plata. C) Micrografia TEM de nanopartículas de magnetita. D) Micrografía SEM de grano de polen de Dimorphotheca ecklonis.

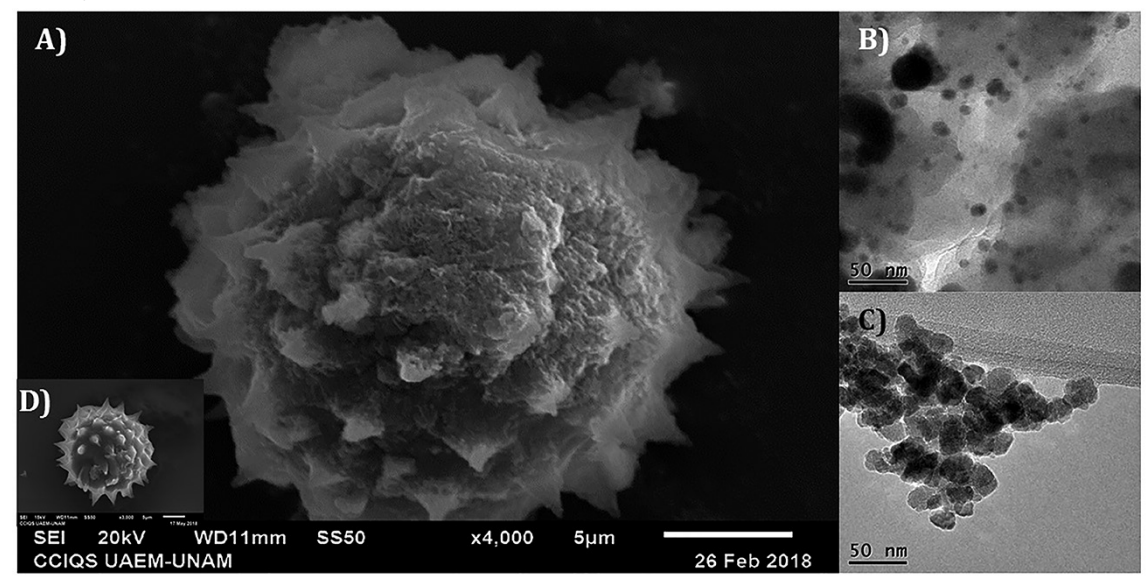

Fuente: Elaboración de los autores.

Ambos tipos de nanopartículas se encuentran distribuidos homogéneamente en el grano de polen como muestra el mapeo elemental por EDS (figura 6). La composición química (\% en peso) determinada con EDS tiene los siguientes valores promedio: $56.82 \%$ carbono, $20.83 \%$ oxígeno, $18.45 \% \mathrm{Fe}$, y, $5.62 \%$ plata.

\section{Conclusiones}

La intensa investigación sobre síntesis verde de nanomateriales ha conseguido resolver el problema del control de tamaño y forma sin perder las ventajas de la biosíntesis. El presente trabajo mostró que es posible obtener diferentes morfologías de nanopartículas de $\mathrm{Au}$ y $\mathrm{Ag}$ por primera vez cuando se utiliza el extracto de Anemopsis californica, así como obtener nanopartícu- 
www.mundonano.unam.mx | ARTículos DE INVESTIGACIón | Mundo Nano

Figura 6. Mapeo elemental EDS de nano/microestructuras híbridas $\mathrm{Ag}-\mathrm{Fe}_{3} \mathrm{O}_{4}$ para los elementos carbono, oxígeno, hierro y plata.
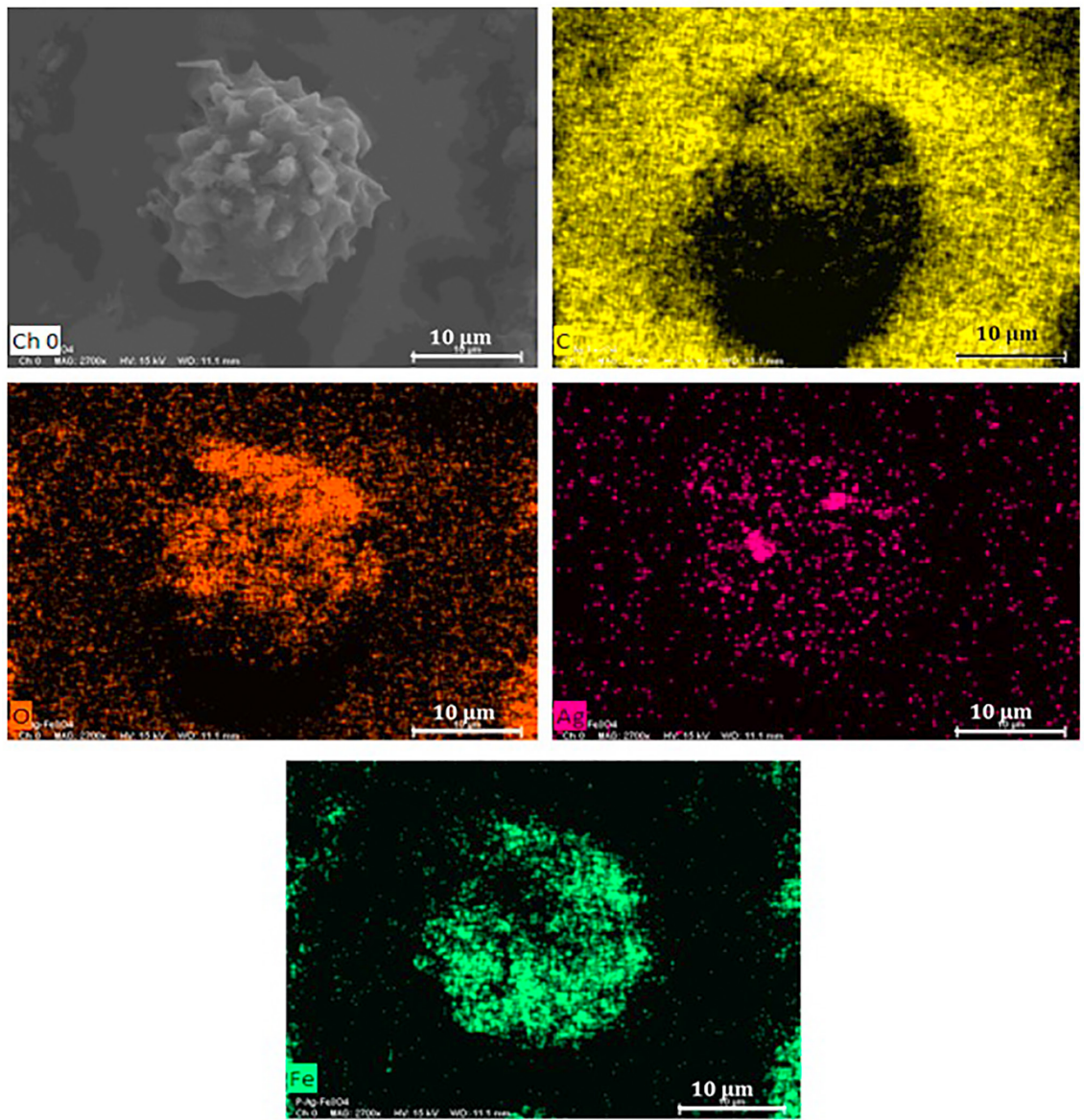

Fuente: Elaboración de los autores.

las de magnetita por biosíntesis en el rango de tamaño de $1.72 \pm 0.38 \mathrm{~nm}$ con un excelente control sobre la fase, utilizando el extracto de Camellia sinensis. A pesar de que aún se requieren estudios profundos sobre el mecanismo involucrado en la síntesis verde de estos sistemas, los resultados mostrados en este artículo y los observados en la literatura revelan que, imitando a la naturaleza, la biosíntesis de nanopartículas se posiciona como un procedimiento con inmenso potencial para la generación de materiales nanoestructurados de forma sencilla, de un mínimo impacto ambiental y ahora con un buen control sobre la forma, tamaño y composición. 


\section{Referencias}

Bhattacharya, D. y Gupta, R. K. (2005). Nanotechnology and potential of microorganisms. Critical Reviews in Biotechnology. https://doi.org/10.1080/07388550500361994

Castro-Longoria, E., Vilchis-Nestor, A. R. y Avalos-Borja, M. (2011). Biosynthesis of silver, gold and bimetallic nanoparticles using the filamentous fungus Neurospora crassa. Colloids and Surfaces B: Biointerfaces. https://doi.org/10.1016/j. colsurfb.2010.10.035

Del-Toro-Sánchez, C. L. et al. (2015). Storage effect on phenols and on the antioxidant activity of extracts from Anemopsis californica and inhibition of elastase enzyme. Journal of Chemistry. https://doi.org/10.1155/2015/602136

Gardea-Torresdey, J. L. et al. (2003). Alfalfa sprouts: A natural source for the synthesis of silver nanoparticles. Langmuir. https://doi.org/10.1021/la020835i

Iravani, S. (2011). Green synthesis of metal nanoparticles using plants. Green Chemistry. https://doi.org/10.1039/c1gc15386b

Kelly, K. L. et al. (2003). The optical properties of metal nanoparticles: The influence of size, shape, and dielectric environment. Journal of Physical Chemistry B. https://doi.org/ 10.1021/jp026731y

Kumar, V. y Yadav, S. K. (2009). Plant-mediated synthesis of silver and gold nanoparticles and their applications. Journal of Chemical Technology and Biotechnology. https://doi.org/10.1002/jctb.2023

Loo, Y. Y., Chieng B. W., Nishibuchi M. y Radu S. (2012). Synthesis of silver nanoparticles by using tea leaf extract from Camellia sinensis. International Journal of Nanomedicine. https://doi.org/10.2147/IJN.S33344

López, G. et al. (2013). Nanoestructuras metálicas; síntesis, caracterización y aplicaciones. Libro de Editorial Reverté, Universidad Autónoma del Estado de México, 1-50.

Medina, A. L. et al. (2005) Composition and antimicrobial activity of Anemopsis californica leaf oil. Journal of Agricultural and Food Chemistry. https://doi.org/10.1021/ jf0511244

Mohanpuria, P., Rana, N. K. y Yadav, S. K. (2008). Biosynthesis of nanoparticles: Technological concepts and future applications. Journal of Nanoparticle Research, 10(3): 507-517. https://doi.org/10.1007/s11051-007-9275-x

Pileni, M. P. (2007). Control of the size and shape of inorganic nanocrystals at various scales from nano to macrodomains. Journal of Physical Chemistry C. https://doi.org/10.1021/jp070646e

Quester, K., Avalos-Borja, M. y Castro-Longoria, E. (2013). Biosynthesis and microscopic study of metallic nanoparticles. Micron. https://doi.org/10.1016/j. micron.2013.07.003

Rababah, T. M., Hettiarachchy, N. S. y Horax, R. (2004). Total phenolics and antioxidant activities of fenugreek, green tea, black tea, grape seed, ginger, rosemary, gotu kola, and ginkgo extracts, vitamin E, and tert-butylhydroquinone. Journal of Agricultural and Food Chemistry. https://doi.org/10.1021/jf049645z

Sajanlal, P. R. et al. (2011). Anisotropic nanomaterials: structure, growth, assembly, and functions. Nano Reviews. https://doi.org/10.3402/nano.v2i0.5883 
Sánchez-Mendieta, V. y Rafael, A. (2012). Green Synthesis of Noble Metal (Au, Ag, Pt) Nanoparticles, Assisted by Plant-Extracts. Noble Metals. https://doi. org/10.5772/34335

Sau, T. K. y Rogach, A. L. (2010). Nonspherical noble metal nanoparticles: Colloidchemical synthesis and morphology control. Advanced Materials. https://doi. org/10.1002/adma.200901271

Schmid, G. (1992). Large clusters and colloids. Metals in the embryonic state Chem Rev. https://doi.org/10.1002/9783527616077

Scott, R. J. (1994). Pollen exine-the sporopollenin enigma and the physics of pattern. Seminar series-society for experimental biology. Cambridge University Press, 49.

Shebanova, O. N. y Lazor, P. (2003). Raman study of magnetite $\left(\mathrm{Fe}_{3} \mathrm{O}_{4}\right)$ : laser induced thermal effects and oxidation. Journal of Raman Spectroscopy. Wiley Online Library, 34(11): 845-852. https://doi.org/10.1002/jrs.1056

Silva-De Hoyos, L. E. et al. (2019). Plasmonic sensing of aqueous-divalent metal ions by biogenic gold nanoparticles. Journal of Nanomaterials. https://doi. org/10.1155/2019/9846729

Vilchis-Néstor, A. R. et al. (2008). Solventless synthesis and optical properties of Au and Ag nanoparticles using Camellia sinensis extract. Materials Letters, 62(1718): 3103-3105. https://doi.org/10.1016/j.matlet.2008.01.138

Xia, Y. et al. (2013). Shape-controlled synthesis of metal nanocrystals. MRS Bulletin. https://doi.org/10.1557/mrs.2013.84

Yoosaf, K. Binil Itty Ipe, Cherumuttathu H. Suresh, K. George Thomas. (2007). In situ synthesis of metal nanoparticles and selective naked-eye detection of lead ions from aqueous media. Journal of Physical Chemistry C. https://doi. org/10.1021/jp073923q 
